\section{BMJ Paediatrics Open}

\title{
Effect of the Fukushima earthquake on weight in early childhood: a retrospective analysis
}

Atsushi Ono, ${ }^{1}$ Tsuyoshi Isojima, ${ }^{2}$ Susumu Yokoya, ${ }^{3}$ Noriko Kato, ${ }^{4}$ Toshiaki Tanaka, ${ }^{5}$ Zentaro Yamagata, ${ }^{6}$ Shoichi Chida, ${ }^{7}$ Hiroko Matsubara, ${ }^{8}$ Soichiro Tanaka, ${ }^{9}$ Mami Ishikuro, ${ }^{10,11}$ Masahiro Kikuya, ${ }^{10,11}$ Shinichi Kuriyama, ${ }^{8,10,11}$ Shigeo Kure, ${ }^{9,10}$ Mitsuaki Hosoya ${ }^{1}$

To cite: Ono A, Isojima T, Yokoya S, et al. Effect of the Fukushima earthquake on weight in early childhood: a retrospective analysis. BMJ Paediatrics Open 2017;2:e000229. doi:10.1136/ bmjpo-2017-000229

- Additional material is published online only. To view please visit the journal online (http://dx.doi.org/10.1136/ bmjpo-2017-000229).

Received 6 November 2017 Revised 5 January 2018 Accepted 14 January 2018
Check for updates

For numbered affiliations see end of article.

\section{Correspondence to}

Dr. Atsushi Ono, Department of Pediatrics, School of Medicine, Fukushima Medical University, Fukushima 960-1295, Japan; hypermowmow@yahoo.co.jp

\section{ABSTRACT}

Objective There have been no reports evaluating the physical growth in early childhood in Fukushima Prefecture after the Great East Japan Earthquake. We retrospectively investigated the health examination data in early childhood (aged 0-3 years).

Methods We divided the affected children into respective groups according to the interval from the disaster to the time of health examination and age as follows: group I, birth to 3-4 months in boys (1.81 (range, 0-6 months)) and girls (1.79 (range, 0-7 months)); group II, 3-4 months to 6-10 months in boys (6.37 (range, 3-9 months)) and girls (6.35 (range, 3-9 months)); group III, 6-10 months and 18 months in boys (16.2 (range, 5-22 months)) and girls (16.9 (range, 5-22 months)); and group IV, 18 months to 36-42 months in boys (21.0 (range, 18-24 months)) and girls (21.0 (range, 18-24 months)). Using height and body mass index, the health status of each group was compared with that of unaffected controls (ie, children who experienced the disaster after their health examination at 36-42 months).

Results The change in body mass index between the health examinations at 18 months and 36-42 months was significantly increased in group I $(95 \% \mathrm{Cl}$ : all boys, 0.192 to 0.276 vs -0.006 to $0.062, P<0.001$ and all girls, 0.108 to 0.184 vs -0.109 to $-0.035, P<0.001$ ) and group II (95\% Cl: all boys, 0.071 to 0.141 vs -0.006 to 0.062 , $\mathrm{P}=0.002$ and all girls, -0.042 to 0.024 vs -0.109 to $-0.035, \mathrm{P}=0.013)$.

Conclusions Children who were affected by the disaster in Fukushima Prefecture in early childhood were overweight. The use of pre-existing information, such as health examination data, was beneficial for investigating the physical growth of affected children.

\section{INTRODUCTION}

The Great East Japan Earthquake occurred on 11 March $2011^{1}$ and caused a great deal of damage in the areas of Iwate, Miyagi and Fukushima Prefectures. ${ }^{2}$ The resultant tsunami destroyed many houses on the Pacific coast and forced many people in the area to evacuate. Moreover, the Fukushima Daiichi Nuclear Power Plant accident forced

\section{What is already known on this topic?}

After the 2011 Great East Japan Earthquake, lifestyle diseases, such as obesity, in affected adults were reported. Recently, overweight in nursery school children aged over 3 years and who were affected by the disaster has also been reported.

\section{What this study hopes to add?}

After the disaster in Fukushima Prefecture, a trend towards being overweight was observed in early childhood (aged 1 to 3 years). The use of pre-existing data on early childhood, such as health examination data, was beneficial for investigating the physical growth of the affected children.

many people living around the power plant to leave their homes. Therefore, the residents in Fukushima Prefecture were likely influenced, either directly or indirectly, by the earthquake disaster. The influence of the disaster on affected adults and nursery school children over the age of 3 years has been reported..$^{3-9}$ In this study, we performed a growth survey on children who were affected in early childhood (aged 0-3 years) in Fukushima Prefecture.

Generally, body mass index (BMI) is used to assess physical data, such as obesity, in adults. However, BMI cannot be compared among children because it varies in different age groups and changes substantially with age. In order to standardise the height and BMI in childhood according to sex and age, we used the SD score (SDS).

\section{METHODS}

Study design and subjects

The survey design and population were described in detail in a previous paper. ${ }^{10}$ 

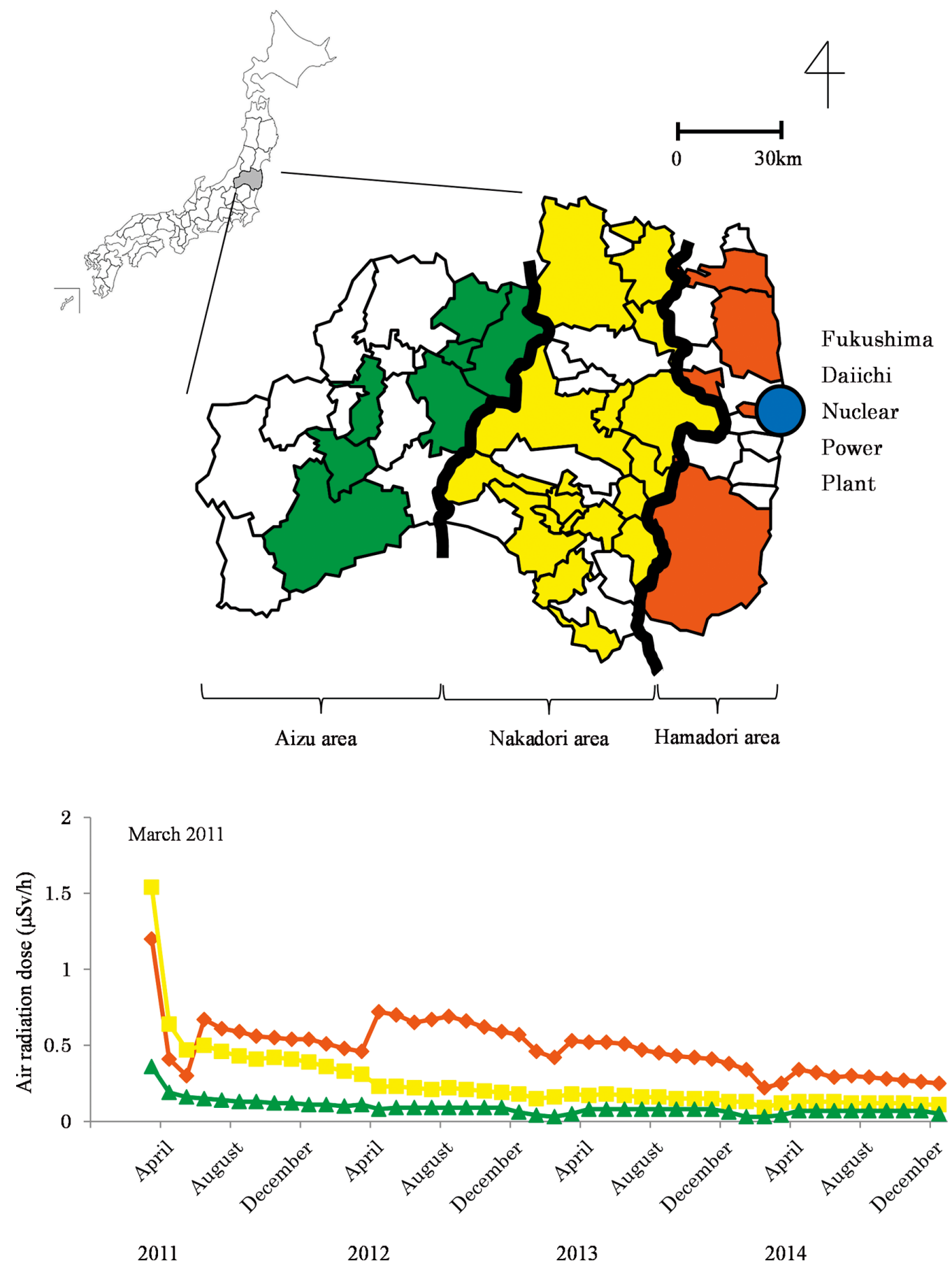

Figure 1 Upper: the 31 local governments that participated in this survey are shown on the map of Fukushima Prefecture. Lower: the air radiation dose from March 2011 to December 2014 for each of the three areas is shown. $\bullet$ : Hamadori area, Nakadori area, $\boldsymbol{\Delta}$ : Aizu area.

According to the Maternal and Child Health Act, local governments across Japan must perform health examinations between 18 months and 2 years and between 3 years and 4 years of age. ${ }^{11}$ Moreover, publicly funded infant medical examinations are carried out in Fukushima Prefecture at 3-4 and 6-10 months of age. We sought to retrospectively investigate the records of these five health examinations, including those at birth, and invited all local governments in Fukushima Prefecture to participate in the survey. Eventually, 31 of 57 local governments, representing $79.6 \%$ of the total number of births in Fukushima Prefecture during the target periods, participated in this survey (figure 1).

Data were provided by the one of the following methods: (1) completion of survey sheets by public health nurses who were employed by the local governments, (2) visits to local health centres to transcribe health examination data or (3) submission of electronic datasets of pre-existing health examination records from which all personally identifiable information was removed. The survey 


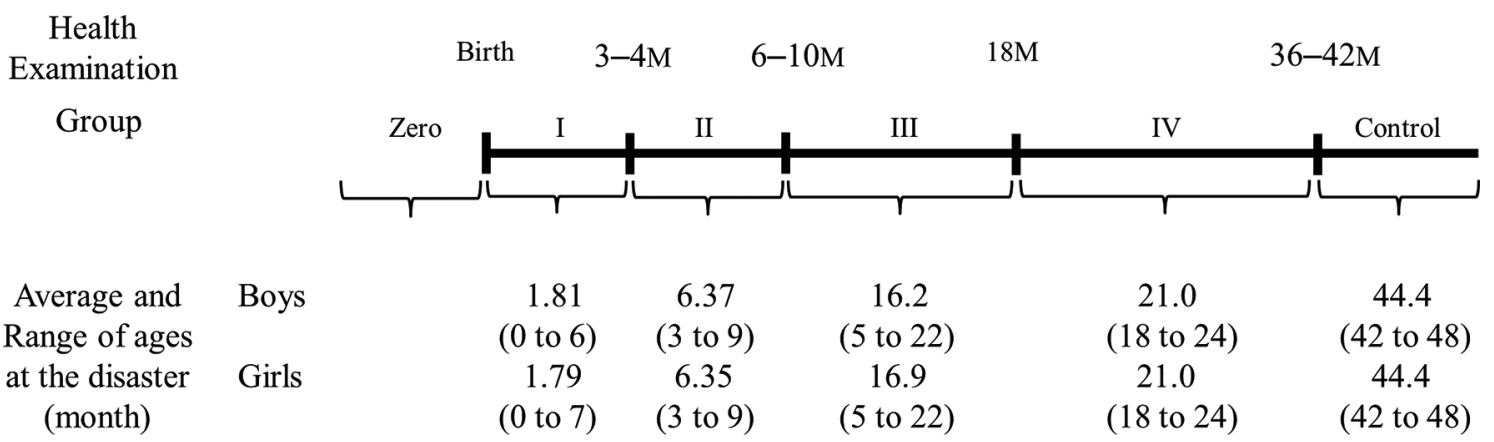

Figure 2 The health examination periods (birth, 3-4 months, 6-10 months, 18 months and 36-42 months) and the six groups (zero, I, II, III, IV and control) divided according to the age during the disaster and average (range) age of each group. M, month health examination.

was conducted from July 2012 to October 2014, in order to collect data on children who were born over a relatively wide range of birth years.

During the periods of investigation, we collected data on 4387 children who were born between 1 March 2007 and 31 August 2007 and who experienced the disaster and its after effects after their health examinations at 36-42 months; on 6167 children who were born between 1 March 2009 and 31 August 2009 and experienced the disaster before their health examinations at 36-42 months; and on 10046 children who were born between 1 June 2010 and 30 April 2011 and who either experienced the disaster when they were under 10 months of age or were not yet born. During these target periods, data were collected from a total of 20600 children, who accounted for $81.9 \%$ of the 25148 total number of births registered by the 31 local governments.

We classified the children into the following six groups: (1) group zero included children who experienced the disaster before birth; (2) group I included children who experienced the disaster between birth and their health

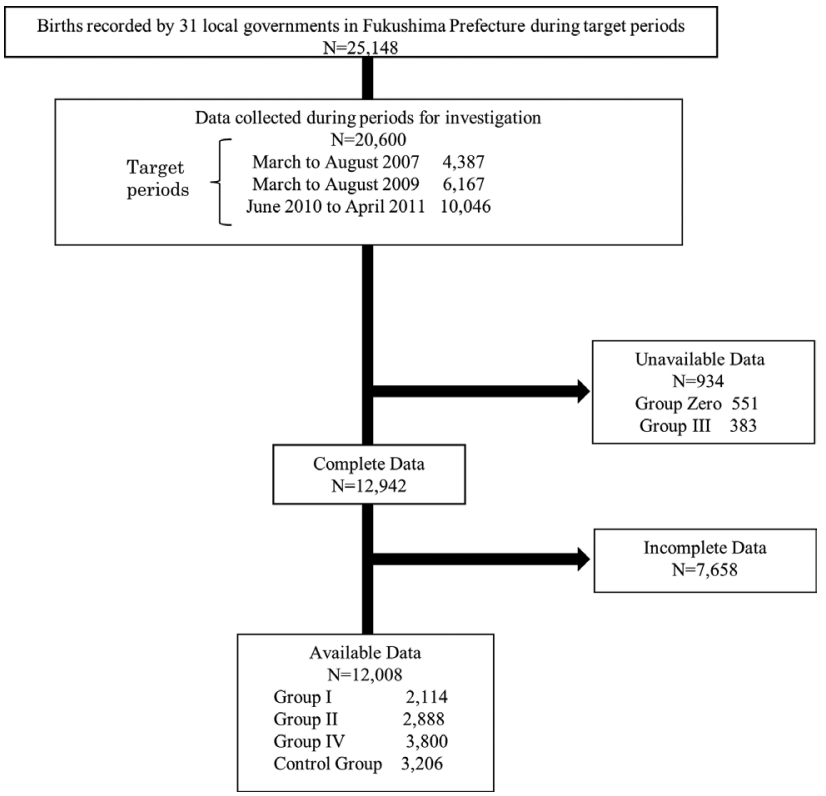

Figure 3 The number of data collected from the 31 local governments participating in this survey. examination at 3-4 months; (3) group II included children who experienced the disaster between their health examinations at 3-4 and 6-10 months; (4) group III included children who experienced the disaster between their health examinations at 6-10 and 18 months; (5) group IV included children who experienced the disaster between their health examinations at 18 and 36-42 months; and (6) the control group, which included 3206 children who experienced the disaster after their health examinations at 36-42 months.

We aimed to compare the groups based on the age when the children experienced the disaster and their physical activity level (figure 2). In the process of gathering such necessary data (figure 3) and in order to ensure that the data gathered were from the same children in all health examinations, we used only complete data from at least four health examinations and excluded the incomplete data. Group zero represented the group of children who experienced the disease before birth and were, therefore, not eligible and excluded from this survey. The number of children who satisfied the definition of group III during the first data collection was very few. Because of this small number and the large difference in the populations, group III was not comparable with the other groups under equal conditions and was excluded. We eventually used the available data from 12008 children.

From the Pacific coast side to the west, Fukushima Prefecture is separated into three areas (ie, Hamadori, Nakadori and Aizu) by mountains and highlands that are 500-2000 $\mathrm{m}$ above sea level. The distance from the west side of the Fukushima Daiichi Nuclear Power Plant to the Hamadori, Nakadori and Aizu areas is approximately $0-85 \mathrm{~km}, 15-100 \mathrm{~km}$ and $70-170 \mathrm{~km}$, respectively. It was thought that the degree of actual radioactive contamination differed among the three areas (figure 1), and the influence on the residents of Fukushima Prefecture was likewise expected to differ. Therefore, we further divided each of the four patient groups according to these three areas and evaluated the corresponding influence of the disaster (table 1 and online supplementary tables S1-S6).

\section{Statistical analysis}

We assessed height and BMI using the records at birth and each of the health examinations undertaken at 3-4 
Table 1 The sex distribution in each area and group in those with complete data

\begin{tabular}{|c|c|c|c|c|c|c|c|}
\hline & \multirow[b]{2}{*}{ Sex } & \multicolumn{6}{|c|}{ Complete data } \\
\hline & & Zero & $\mathbf{I}$ & II & III & IV & Control \\
\hline \multirow[t]{2}{*}{ All area $\dagger$} & Boys & 297 & (836)1061 & $(1174) 1478$ & 180 & (1492)1911 & (1414)1645 \\
\hline & Girls & 254 & (843)1053 & $(1121) 1410$ & 203 & (1436)1889 & (1333)1561 \\
\hline \multirow[t]{2}{*}{ Hamadori area } & Boys & 145 & 351 & 460 & 26 & 573 & 477 \\
\hline & Girls & 115 & 328 & 420 & 32 & 558 & 392 \\
\hline \multirow[t]{2}{*}{ Nakadori area } & Boys & 118 & $(330) 555$ & $(505) 809$ & 129 & (688)1107 & (732)963 \\
\hline & Girls & 116 & $(371) 581$ & $(508) 797$ & 144 & $(680) 1133$ & (730)958 \\
\hline \multirow[t]{2}{*}{ Aizu area } & Boys & 34 & 155 & 209 & 25 & 231 & 205 \\
\hline & Girls & 23 & 144 & 193 & 27 & 198 & 211 \\
\hline
\end{tabular}

*The only data from group I, II, IV and control are used in this study.

†The number in brackets represents the number of children who were examined at birth. They are fewer than the number of other health examination periods because some data on height at birth were missing in Nakadori area.

months, 6-10 months, 18 months and 36-42 months of age. Due to the fact that BMI in childhood changes substantially with age, comparison of BMI among children of different age groups is difficult. Moreover, the age in months of children taking the same health examination varies. For that reason, it was necessary to standardise each BMI in childhood according to sex and age. Cole ${ }^{12}$ constructed centile curves for BMI using the lambda-musigma (LMS) method, which was adopted by Inokuchi et al for the Japanese population. ${ }^{13}$ Therefore, we are now able to express BMI as SDS. SD is defined as the square root of the variance and represents the width of the distribution. The zero of SDS represents the average value, and the SDS represents the number of times that the SD diverges from the average value. We converted the height and BMI of all children to height SDS and BMI SDS using a calculation software, ${ }^{14}$ and then calculated the $\triangle \mathrm{BMI}$ SDS as the difference in BMI SDS between two consecutive health examinations as follows: between birth and 3-4 months, between 3-4 and 6-10 months and between 18 and $36-42$ months.

Using the Microsoft Excel 2010 software package for Windows, the t-test was performed to assess the significance of differences in height SDS, BMI SDS and $\triangle \mathrm{BMI}$ SDS. We stratified the indicators by region and sex in order to examine the differences between the control group and groups I, II and IV. In our analysis, because there were three pairwise comparisons between the control group and the other three groups for each analysis stratified by region and sex, a $\mathrm{P}$ value of less than 0.016 was regarded as statistically significant using the Bonferroni correction.

\section{Ethics committee approval}

Our survey was conducted in accordance with the National Ethical Guidelines for Epidemiological Research. ${ }^{15}$ We collected only existing data and, therefore, did not obtain informed consent from the participants. Accordingly, we disclosed information, including the significance, objectives and methods of the survey, to the public via the website of the School of Medicine, Tohoku University (http://www.med.tohoku.ac.jp/ public/ekigaku2013.html).

\section{RESULTS}

We compared the height SDS, BMI SDS and $\triangle$ BMI SDS before and after the disaster between the affected children (group I, II and IV) and the unaffected children (control group). Moreover, we compared the $\triangle$ BMI SDS in these groups according to the three areas (ie, Hamadori, Nakadori and Aizu).

\section{Height SDS}

As shown in figure 4 and table 2, no significant differences were observed between the control group and groups I, II and IV.

\section{BMI SDS}

As shown in figure 5 and table 3 , the BMI SDS of groups I, II and IV was equal to or less than that of the control group before the disaster. After the disaster, on the health examination at 36-42 months, the BMI SDS for boys in the control group (95\% CI 0.187 to 0.277 ) was significantly lower than that in group I (95\% CI 0.298 to $0.404 ; \mathrm{P}=0.001)$ and group II $(95 \% \mathrm{CI} 0.302$ to 0.390 ; $\mathrm{P}<0.001)$. For girls, the BMI SDS in the control group (95\% CI:0.162 to 0.250 ) was significantly lower than that in group I (95\% CI 0.240 to $0.346 ; \mathrm{P}=0.013$ ) and group II (95\% CI 0.265 to $0.355 ; \mathrm{P}=0.001$ ).

\section{$\triangle B M I$ SDS}

As shown in figure 6 and table 4 , in the Hamadori area, the $\triangle$ BMI SDS between the health examinations at 3-4 and 6-10 months for boys was higher in group I than in the control group (95\% CI 0.085 to 0.233 vs -0.124 to 0.030 , $\mathrm{P}<0.001)$. The $\triangle \mathrm{BMI}$ SDS between the health examinations at 18 months and 36-42 months was significantly higher in group I than in the control group in both boys (95\% CI 0.319 to 0.473 vs -0.052 to $0.068, \mathrm{P}<0.001$ ) and 

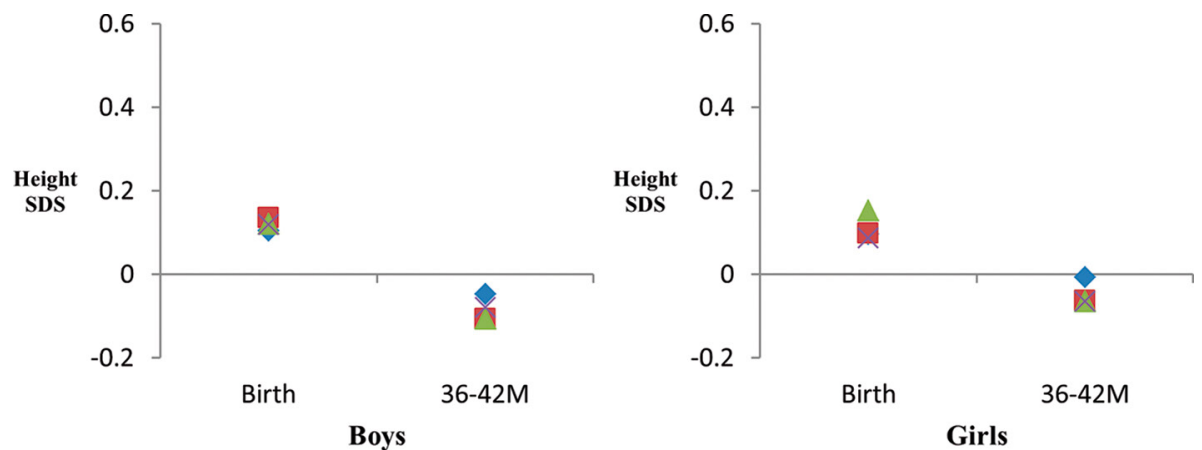

Figure 4 Height SDS of all boys and girls in Fukushima Prefecture. month health examination; SDS, SD score.

girls $(95 \%$ CI 0.208 to 0.342 vs -0.206 to $-0.048, \mathrm{P}<0.001)$. The $\triangle$ BMI SDS between the health examinations at 18 months and 36-42 months was significantly higher in group II than in the control group in both boys $(95 \% \mathrm{CI}$ 0.163 to 0.285 vs -0.052 to $0.068, \mathrm{P}<0.001$ ) and girls (95\% CI 0.019 to 0.148 vs -0.206 to $-0.048, \mathrm{P}<0.001$ ).

In the Nakadori area, the $\triangle \mathrm{BMI}$ SDS between the health examinations at 18 months and 36-42 months was significantly higher in group I than in the control group in both boys $(95 \%$ CI 0.123 to 0.233 vs 0.008 to $0.100, \mathrm{P}=0.001$ ) and girls (95\% CI 0.062 to 0.166 vs -0.086 to 0.004 , $\mathrm{P}<0.001)$. For all of the three periods in the Aizu area, no significant differences were observed between the control group and groups I, II and IV.

\section{DISCUSSION}

The $\triangle$ BMI SDS between the health examinations at 18 months and 36-42 months was significantly increased in children who experienced the disaster between birth and their health examination at $6-10$ months. Also, a trend towards overweight children was observed in the Hamadori and Nakadori areas, but not in the Aizu area.

In the aftermath of the tsunami, Fukushima Prefecture was severely affected by the Fukushima Daiichi Nuclear
Power Plant accident. The radiation levels were relatively high in the Hamadori area, somewhat elevated in the Nakadori area and only slightly elevated in the Aizu area (figure 1) ${ }^{16}$ After the accident, the Ministry of Education, Culture, Sports, Science and Technology and the Ministry of Health, Labour and Welfare of Japan jointly released a statement on restrictions to outdoor activity in all schools and nursery schools based on the air radiation. Restrictions on outdoor activity were enforced by the local governments across almost all of the Hamadori area and much of the Nakadori area that had high radiation levels. ${ }^{17} 18$

There was a relatively close correlation between the areas with high air radiation level in which restriction of outdoor activity was deemed necessary and the areas in which a trend towards overweight children was observed. The impact of restriction of outdoor activity after the disaster might have been more significant on children aged over 1 year than on other children probably because of the fact that toddlers are generally encouraged and actually begin to engage in various activities, including outdoor play, after 1 year of age. ${ }^{19}$

Body mass in young adults is strongly related to body mass in childhood. ${ }^{20}$ Moreover, an early adiposity

Table 2 Number, average $(95 \% \mathrm{Cl})$ and P value for height SDS in Fukushima Prefecture

\begin{tabular}{|c|c|c|c|c|c|c|}
\hline \multirow[b]{2}{*}{ Height SDS } & \multicolumn{3}{|l|}{ Birth } & \multicolumn{3}{|c|}{$36-42 M$} \\
\hline & $\mathbf{n}$ & Average $(95 \% \mathrm{Cl})$ & $P$ value & $\mathbf{n}$ & Average (95\% Cl) & $P$ value \\
\hline \multicolumn{7}{|l|}{ All boys } \\
\hline Group I & 836 & $0.136(0.065$ to 0.207$)$ & 0.522 & 1061 & $-0.106(-0.164$ to -0.048$)$ & 0.110 \\
\hline Group II & 1174 & 0.121 (0.064 to 0.178$)$ & 0.728 & 1478 & $-0.107(-0.154$ to -0.060$)$ & 0.069 \\
\hline \multicolumn{7}{|l|}{ All girls } \\
\hline Control group & 1333 & 0.097 (0.038 to 0.156$)$ & & 1561 & $-0.006(-0.055$ to 0.043$)$ & \\
\hline Group I & 843 & 0.098 (0.025 to 0.171$)$ & 0.979 & 1053 & $-0.063(-0.122$ to -0.004$)$ & 0.143 \\
\hline Group II & 1121 & 0.153 (0.096 to 0.210$)$ & 0.187 & 1410 & $-0.065(-0.115$ to -0.015$)$ & 0.100 \\
\hline
\end{tabular}

$\mathrm{M}$, month health examination; SDS, SD score. 

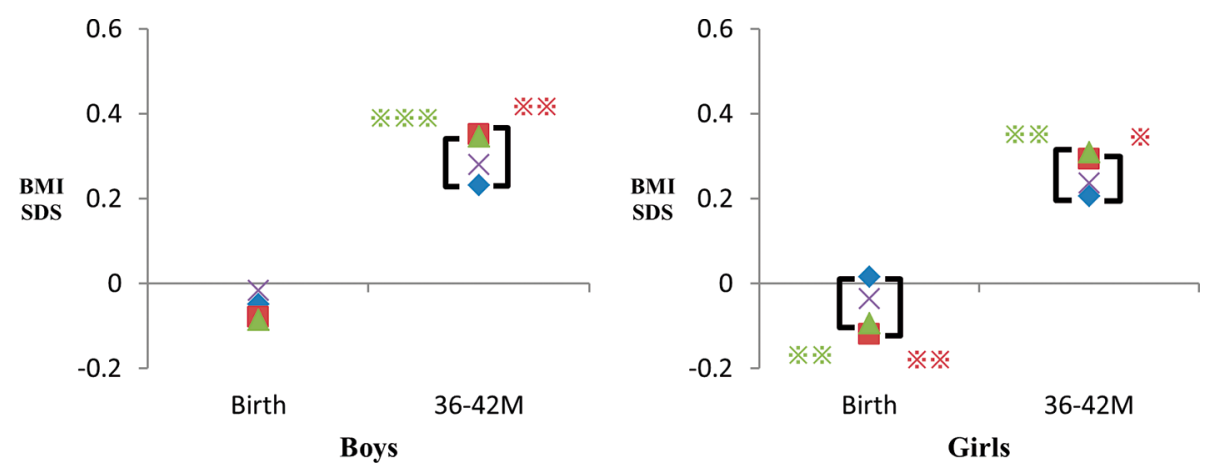

Figure 5 The BMI SDS of all boys and girls in Fukushima Prefecture. $\bullet$ : control group, $\mathbf{\square}$ : group I, $\mathbf{\Delta}$ : group II, $\mathbf{x}$ : group IV. *P value $<0.016,{ }^{* \star} \mathrm{P}$ value $<0.01,{ }^{* \star *} \mathrm{P}$ value $<0.001$. BMI, body mass index; $\mathrm{M}$, month health examination; SDS, SD score.

rebound has been found to be significantly associated with BMI level at a later age and an increased risk for being overweight. ${ }^{21}{ }^{22}$ Early detection of adiposity rebound is important in preventing metabolic syndrome. ${ }^{23}$ It is also important to promote early intervention in order to prevent adult obesity. Some studies have reported that stress on the affected mothers caused growth failure in children during the perinatal period ${ }^{24} 25$ and is a risk factor for childhood obesity. ${ }^{26}$ Early intervention for the affected mothers prior to delivery is, therefore, also necessary to preserve the health of their children. ${ }^{27}$

There are three limitations to this study. First, we used data from only 31 of 57 local governments that agreed to participate in this survey. Nevertheless, these local governments were large municipalities and covered $79.6 \%$ of the number of childbirths in Fukushima Prefecture during the target periods. Second, we did not investigate the causes of overweight in detail and outdoor activity itself in early childhood; there had been no comprehensive or extensive survey on outdoor activity in early childhood after the disaster. Being overweight may be associated with an unbalanced diet; the changes in the eating habits in the evacuation area were considered to be one of the causes of adult obesity. ${ }^{528}$ However, there are no reports on the relationship between early childhood diet, such as milk or baby food, and changes in body size before and after the disaster. Another possible cause of overweight is stress, and it has been shown that stress may cause growth failure and lead to being overweight. Sleep disorders may also be an important factor in being overweight among affected adults and children. ${ }^{29}{ }^{30}$ However, we did not investigate in this study the influence of stress and sleep disorders on being overweight. Therefore, diet, stress and sleep disorders cannot be excluded as factors that might have contributed to being overweight in this study. Lastly, because the interval between data collections was more than 6 months, the number of children included in group III became much smaller than the other groups. Consecutive data collection could have minimised the difference between sample sizes of the groups analysed.

We retrospectively analysed the data on health examination and discovered that the availability of normal baseline data for comparison with those after the event were useful. The health examination data from within Fukushima prefecture is large, but not all could be obtained and the only common variables available were height and weight. The use of an online backup service and standardisation of the format of data sheets to manage health examination records nationwide will likely enable easier collection of more data.

Table 3 Number, average $(95 \% \mathrm{Cl})$ and $\mathrm{P}$ value for BMI SDS in Fukushima Prefecture

\begin{tabular}{|c|c|c|c|c|c|c|}
\hline \multirow[b]{2}{*}{ BMI SDS } & \multicolumn{3}{|l|}{ Birth } & \multicolumn{3}{|c|}{ 36-42 M } \\
\hline & $\mathbf{n}$ & Average $(95 \% \mathrm{Cl})$ & $P$ value & $\mathbf{n}$ & Average $(95 \% \mathrm{Cl})$ & $P$ value \\
\hline \multicolumn{7}{|l|}{ All boys } \\
\hline Group I & 836 & $-0.080(-0.146$ to -0.014$)$ & 0.479 & 1061 & 0.351 (0.298 to 0.404$)$ & 0.001 \\
\hline Group II & 1174 & $-0.085(-0.143$ to -0.027$)$ & 0.363 & 1478 & $0.346(0.302$ to 0.390$)$ & $<0.001$ \\
\hline \multicolumn{7}{|l|}{ All girls } \\
\hline Control group & 1333 & $0.016(-0.041$ to 0.073$)$ & & 1561 & $0.206(0.162$ to 0.250$)$ & \\
\hline Group I & 843 & $-0.119(-0.190$ to -0.048$)$ & 0.004 & 1053 & 0.293 (0.240 to 0.346$)$ & 0.013 \\
\hline Group II & 1121 & $-0.094(-0.155$ to -0.033$)$ & 0.009 & 1410 & $0.310(0.265$ to 0.355$)$ & 0.001 \\
\hline
\end{tabular}

BMI, body mass index; M, month health examination; SDS, body mass index SD score. 


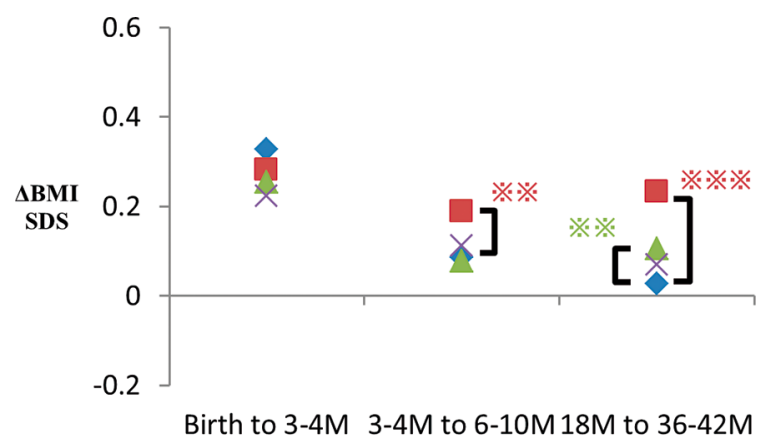

All Boys
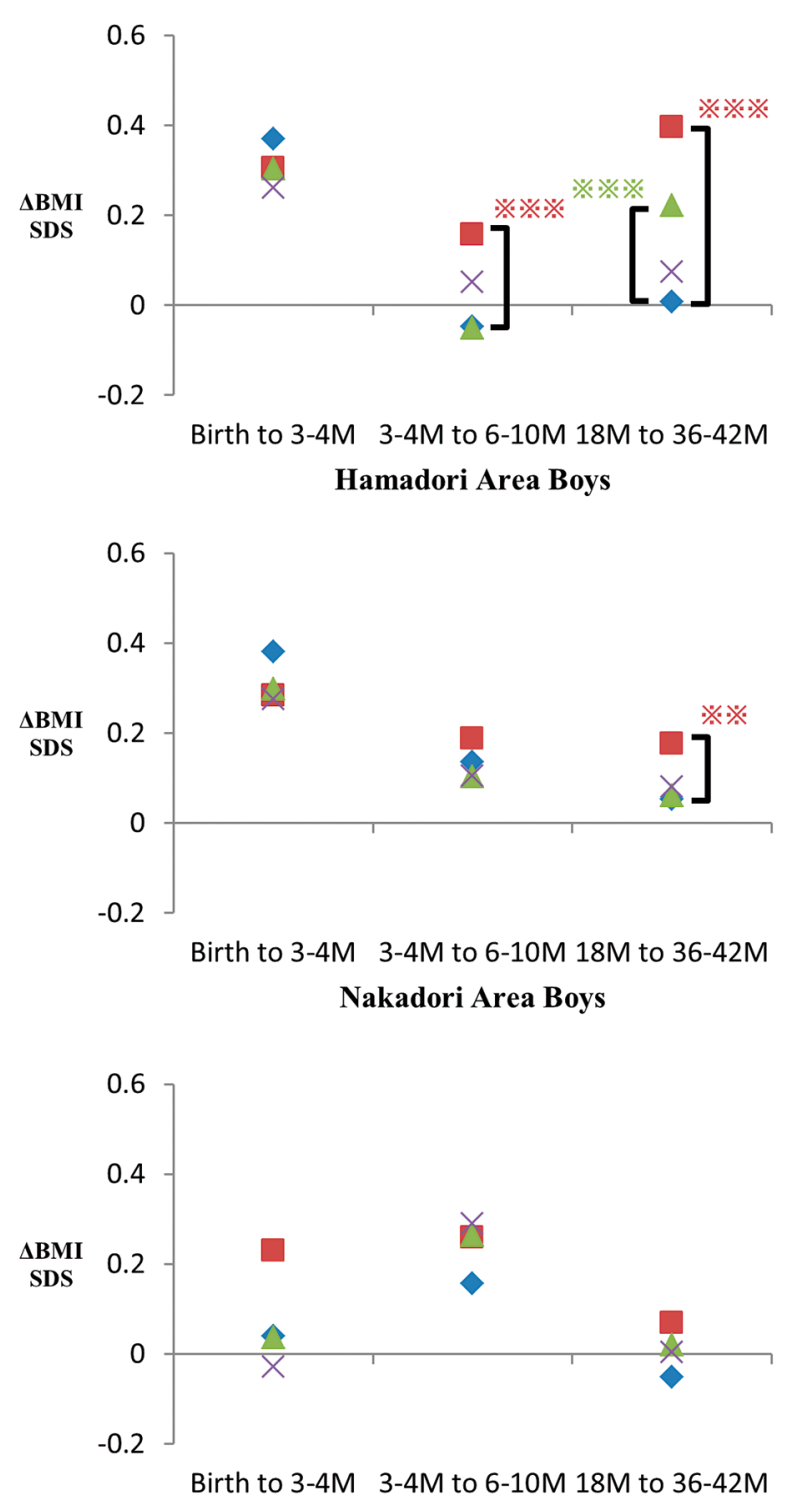

Aizu Area Boys
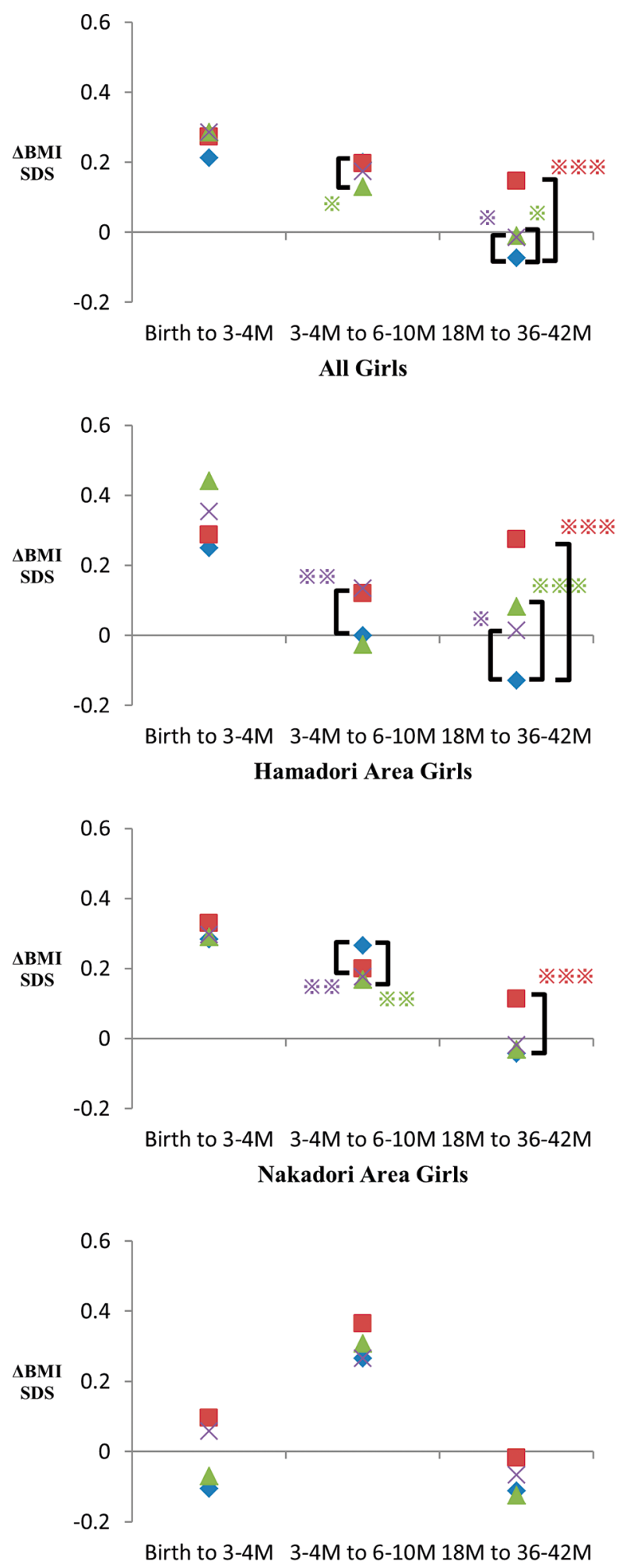

Aizu Area Girls

Figure $6 \quad \triangle B M I$ SDS of all boys and girls in Fukushima Prefecture. $\downarrow$ : control group, $\mathbf{\square}$ : group I, $\mathbf{A}$ : group II, $\times$ : group IV. *P value $<0.016,{ }^{* *} \mathrm{P}$ value $<0.01$, ${ }^{* * *} \mathrm{P}$ value $<0.001$. BMI, body mass index; $\triangle \mathrm{BMI} \mathrm{SDS}$, BMI SDS between two consecutive health examinations; M, month health examination; SDS, SD score.

\section{CONCLUSION}

After the disaster, being overweight was observed between the health examination at 18 months and 36-42 months in children who experienced the disaster between birth and their health examination at 6-10 months; this trend was observed in the Hamadori and Nakadori areas where the radiation levels were relatively high. The use of pre-existing health 


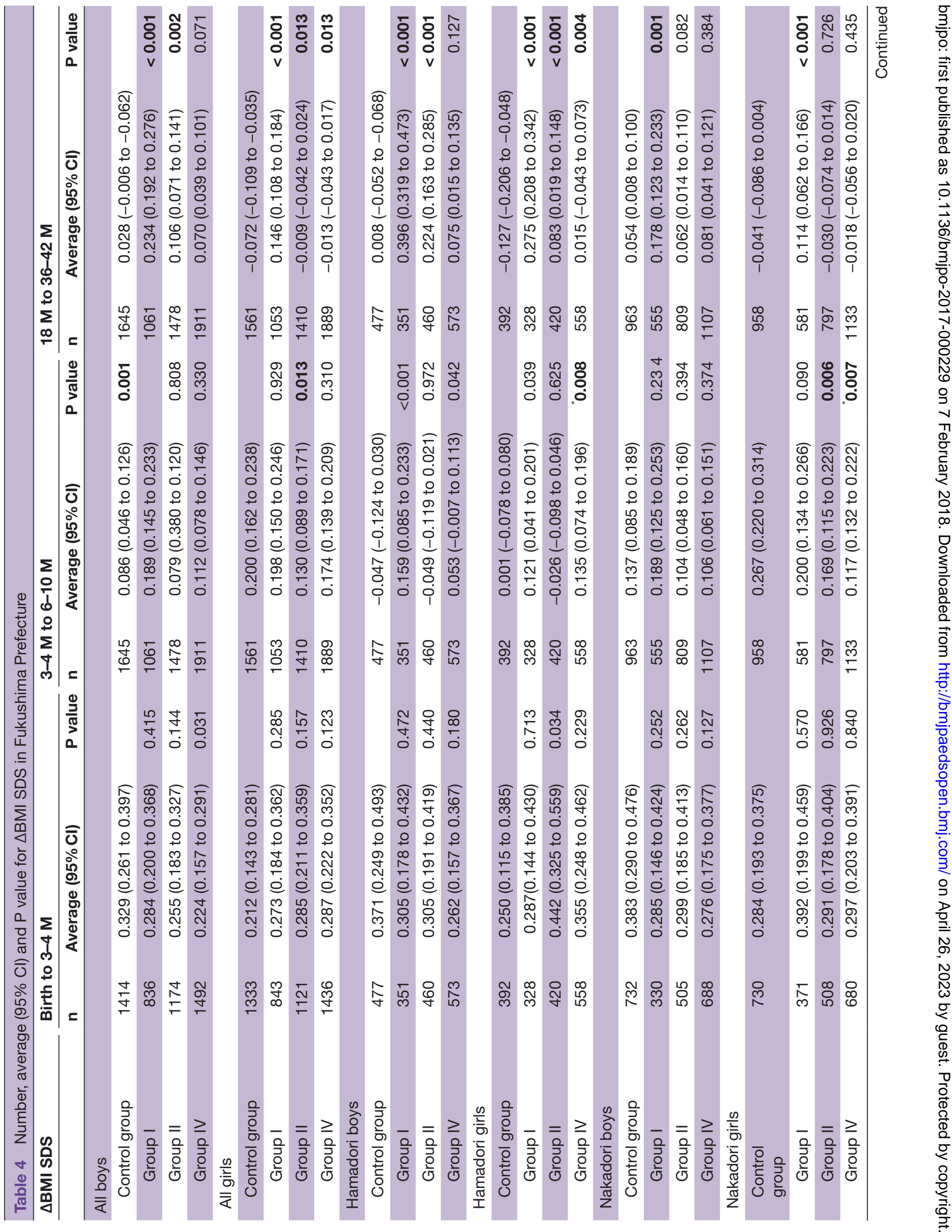


examination data in early childhood, when physical growth was marked, was beneficial because the physical examination findings were described in detail.

Author affiliations

${ }^{1}$ Department of Pediatrics, School of Medicine, Fukushima Medical University, Fukushima, Japan

${ }^{2}$ Department of Pediatrics, Graduate School of Medicine, The University of Tokyo, Tokyo, Japan

${ }^{3}$ Department of Medical Subspecialties, National Center for Child Health and Development, Tokyo, Japan

${ }^{4}$ Department of Early Childhood and Elementary Education, Jumonji University, Niiza, Japan

${ }^{5}$ Japanese Association for Human Auxology, Tokyo, Japan

${ }^{6}$ Department of Health Sciences, Interdisciplinary Graduate School of Medicine and Engineering, University of Yamanashi, Chuo, Japan

${ }^{7}$ Department of Pediatrics, School of Medicine, Iwate Medical University, Morioka, Japan

${ }^{8}$ Department of Disaster Public Health, International Research Institute of Disaster Science (IRIDeS), Tohoku University, Sendai, Japan

${ }^{9}$ Department of Pediatrics, Graduate School of Medicine, Tohoku University, Sendai, Japan

${ }^{10}$ Tohoku Medical Megabank Organization (ToMMo), Tohoku University, Sendai, Japan

${ }^{11}$ Department of Molecular Epidemiology, Graduate School of Medicine, Tohoku University, Sendai, Japan

Contributors A0 collected, analysed and interpreted the data, and drafted the initial manuscript. SK conceptualised and designed the study, obtained funding, and analysed and interpreted the data. SY, NK, TT, ZY, SC, ST, MK and SK conceptualised and designed the study, and analysed and interpreted the data. TI, $\mathrm{HM}$ and $\mathrm{MI}$ collected, analysed and interpreted the data. MH conceptualised and designed the study, analysed and interpreted the data, and reviewed and revised the manuscript. All authors approved the final manuscript submitted and agree to be accountable for all aspects of the work.

Funding This study was conducted as part of the 'Surveillance Study on Child Health in the Great East Japan Earthquake Disaster Area' and supported in full by funding from the Health and Labour Sciences Research Grant (H24-jisedaishitei-007, fukkou).

Competing interests None declared.

Ethics approval The survey protocol was approved by the institutional review board of Fukushima Medical University (authorisation number 1487).

Provenance and peer review Not commissioned; externally peer reviewed. Open Access This is an Open Access article distributed in accordance with the Creative Commons Attribution Non Commercial (CC BY-NC 4.0) license, which permits others to distribute, remix, adapt, build upon this work non-commercially, and license their derivative works on different terms, provided the original work is properly cited and the use is non-commercial. See: http://creativecommons.org/ licenses/by-nc/4.0/

(c) Article author(s) (or their employer(s) unless otherwise stated in the text of the article) 2017. All rights reserved. No commercial use is permitted unless otherwise expressly granted.

\section{REFERENCES}

1. Japan Meteorological Agency. Information on the 2011 great East Japan earthquake. http://www.data.jma.go.jp/svd/eqev/data/2011 03_11_tohoku/ (accessed 20 Apr 2017).

2. National Police Agency of Japan. Damaged situation and police countermeasures associated with 2011 Tohoku district-off the Pacific Ocean Earthquake. https://www.npa.go.jp/archive/keibi/biki/ higaijokyo_e.pdf (accessed 20 Apr 2017).

3. Tsubokura M, Takita M, Matsumura T, et al. Changes in metabolic profiles after the Great East Japan Earthquake: a retrospective observational study. BMC Public Health 2013;13:267.

4. Kawasaki Y, Hosoya M, Yasumura S, et al. Fukushima Health Management Survey Group. The basic data for residents aged 16 years or older who received a comprehensive health check examinations in 2011-2012 as a part of the fukushima health 
management survey after the great East Japan earthquake. Fukushima J Med Sci 2014;60:159-69.

5. Ohira T, Hosoya M, Yasumura S, et al. How lifestyle affects healthchanges in health status before and after the earthquake. Fukushima J Med Sci 2014;60:211-2.

6. Yokomichi H, Zheng W, Matsubara H, et al. Impact of the great east Japan earthquake on the body mass index of preschool children: a nationwide nursery school survey. BMJ Open 2016;6:e010978.

7. Zheng W, Yokomichi $\mathrm{H}$, Matsubara $\mathrm{H}$, et al. Longitudinal changes in body mass index of children affected by the Great East Japan Earthquake. Int J Obes 2017;41:606-12.

8. Kikuya $\mathrm{M}$, Matsubara $\mathrm{H}$, Ishikuro $\mathrm{M}$, et al. Alterations in physique among young children after the Great East Japan Earthquake: Results from a nationwide survey. J Epidemiol 2017;27:462-8.

9. Isojima T, Yokoya S, Ono A, et al. Prolonged elevated body mass index in preschool children after the Great East Japan Earthquake. Pediatr Int 2017;59:1002-9.

10. Matsubara $\mathrm{H}$, Ishikuro M, Kikuya $\mathrm{M}$, et al. Design of the health examination survey on early childhood physical growth in the Great East Japan Earthquake affected areas. J Epidemiol 2017;27:135-42.

11. National Diet Library. Maternal and child health act, law number: act no. 141 of 1965 (in Japanese). http://law.e-gov.go.jp/cgi-bin/ idxselect.cgi?IDX OPT $=1 \& \mathrm{H}$ NAME $=\% 95 \%$ ea $\% 8 \mathrm{e} \% 71 \% 95 \% \mathrm{db} \%$ $8 c \% 92 \& H \_N A M E \_Y O M I=\% 82 \% a 0 \& H \_N O \_G E N G O=H \& H \_N O$ YEAR $=\& H \_N O \_T Y P E=2 \& H \_N O \_N O=\& H \_F I L E \_N A M E=S 40 \mathrm{HO} 141 \&$ H_RYAKU=1\&H_CTG=1\&H_YOMI_GUN=1\&H_CTG_GUN=1 (accessed 20 Apr 2017).

12 Cole TJ. The LMS method for constructing normalized growth standards. Eur J Clin Nutr 1990;44:45-60.

13. Inokuchi M, Hasegawa T, Anzo M, et al. Standardized centile curves of body mass index for Japanese children and adolescents based on the 1978-1981 national survey data. Ann Hum Biol 2006;33:444-53.

14. The Japanese Society of Pediatric Endocrinology. Software for BMI and BMI percentile SDS. http://jspe.umin.jp/medical/taikaku.html (accessed 20 Apr 2017).

15. Ministry of Education, Culture, Sports, Science and Technology, and Ministry of Health, Labour and Welfare. Ethical guidelines for epidemiological research (in Japanese). http://www.lifescience.mext. go.jp/files/pdf/37_139.pdf (accessed 20 Apr 2017).

16. Fukushima Prefecture. Result of monitoring survey of radiation levels in Fukushima Prefecture. https://www.pref.fukushima.lg.jp/sec/ 16025d/kako-monitoring.html (accessed 20 April, 2017).

17. Ministry of Education, Culture, Sports, Science and Technology, Japan. The temporary way of thinking in the use judgements of buildings and grounds of schools in Fukushima. http://www.mext. go.jp/a_menu/saigaijohou/syousai/1305173.htm (accessed $20 \mathrm{Apr}$ 2017).

18. Ministry of Health, Labour and Welfare, Japan. The temporary way of thinking in the use judgements of buildings and grounds of nursery schools in Fukushima. http://www.mhlw.go.jp/stf/houdou/ 2r98520000019qpz-att/2r9852000001nluo.pdf (accessed $20 \mathrm{Apr}$ 2017).

19. Tremblay MS, Leblanc AG, Carson V, et al. Canadian physical activity guidelines for the early years (aged 0-4 years). Appl Physiol Nutr Metab 2012;37:345-56.

20. Tsukada H, Miura K, Kido T, et al. [Relationship of childhood obesity to adult obesity: a 20-year longitudinal study from birth in Ishikawa Prefecture, Japan]. Nihon Koshu Eisei Zasshi 2003;50:1125-34.

21. Rolland-Cachera MF, Deheeger M, Bellisle F, et al. Adiposity rebound in children: a simple indicator for predicting obesity. Am J Clin Nutr 1984;39:129-35.

22. Rolland-Cachera MF, Deheeger M, Maillot M, et al. Early adiposity rebound: causes and consequences for obesity in children and adults. Int J Obes 2006;30(Suppl 4):S11-7.

23. Koyama S, Ichikawa G, Kojima M, et al. Adiposity rebound and the development of metabolic syndrome. Pediatrics 2014;133:e114-19.

24. Harville E, Xiong X, Buekens P. Disasters and perinatal health:a systematic review. Obstet Gynecol Surv 2010;65:713-28.

25. Bromet EJ, Havenaar JM, Guey LT. A 25 year retrospective review of the psychological consequences of the Chernobyl accident. Clin Oncol 2011;23:297-305.

26. Dancause KN, Laplante DP, Fraser S, et al. Prenatal exposure to a natural disaster increases risk for obesity in 51/2-year-old children. Pediatr Res 2012;71:126-31.

27. Goto A, Bromet EJ, Fujimori K. Immediate effects of the Fukushima nuclear power plant disaster on depressive symptoms among mothers with infants: a prefectural-wide cross-sectional study from the Fukushima health management survey. BMC Psychiatry 2015;15:59

28. Inoue T, Nakao A, Kuboyama K, et al. Gastrointestinal symptoms and food/nutrition concerns after the great East Japan earthquake in March 2011: survey of evacuees in a temporary shelter. Prehosp Disaster Med 2014;29:303-6.

29. Ohira T, Hosoya M, Yasumura S, et al. Effect of evacuation on body weight after the great East Japan earthquake. Am J Prev Med 2016:50:553-60.

30. Halal CS, Matijasevich A, Howe LD, et al. Short sleep duration in the first years of life and obesity/overweight at age 4 years: a birth cohort study. J Pediatr 2016;168:99-103. 